\title{
Extensive Genome-wide Linkage Disequilibrium in Cattle
}

\author{
Frédéric Farnir, Wouter Coppieters, Juan-José Arranz, Paulette Berzi, \\ Nadine Cambisano, Bernard Grisart, Latifa Karim, Fabienne Marcq, \\ Laurence Moreau, Myriam Mni, Carine Nezer, Patricia Simon, \\ Pascal Vanmanshoven, Danny Wagenaar, and Michel Georges ${ }^{1}$ \\ Department of Genetics, Faculty of Veterinary Medicine, University of Liège (B43), 4000-Liège, Belgium
}

\begin{abstract}
A genome-wide linkage disequilibrium (LD) map was generated using microsatellite genotypes (284 autosomal microsatellite loci) of 581 gametes sampled from the dutch black-and-white dairy cattle population. LD was measured between all marker pairs, both syntenic and nonsyntenic. Analysis of syntenic pairs revealed surprisingly high levels of LD that, although more pronounced for closely linked marker pairs, extended over several tens of centimorgan. In addition, significant gametic associations were also shown to be very common between nonsyntenic loci. Simulations using the known genealogies of the studied sample indicate that random drift alone is likely to account for most of the observed disequilibrium. No clear evidence was obtained for a direct effect of selection ("Bulmer effect"). The observation of long range disequilibrium between syntenic loci using low-density marker maps indicates that LD mapping has the potential to be very effective in livestock populations. The frequent occurrence of gametic associations between nonsyntenic loci, however, encourages the combined use of linkage and linkage disequilibrium methods to avoid false positive results when mapping genes in livestock.
\end{abstract}

Recently, linkage disequilibrium (LD) has received considerable attention as it may be exploited to more effectively map genes underlying both simple and complex (dichotomous and continuously distributed) traits (Terwilliger and Weiss 1998). The potential advantage of LD mapping over conventional linkage analysis performed within families lies in the use of "historical" recombinants, thereby increasing mapping resolution (e.g., Hästbacka et al. 1992; Talbot et al. 1999) and power. To be effective, however, LD-mapping requires a marker density compatible with the distances across which LD extends in the population of interest. Kruglyak (1999) estimated by simulation that useful levels of LD were unlikely to extend beyond an average distance of $3 \mathrm{~kb}$ in the human, thereby implying the need for a marker map comprising 500,000 SNPs Although experimental LD data are accumulating in the human (e.g., Laan and Pääbo 1997; Nickerson et al. 1998) and some primate species (Crouau-Roy et al. 1996), little is known about the extent of LD in most other mammals, including domestic species. In this paper, we have used genotypes obtained with a panel of 284 microsatellites to measure genome-wide LD in the dutch black-andwhite dairy cattle population. We make the remarkable observation that intrachromosomal LD extends over several tens of centimorgans, and that gametic phase disequilibrium is common between non syntenic loci.

'Corresponding author.

E-MAIL michel.georges@ulg.ac.be; FAX 32043664122.

\section{RESULTS}

Evidence for Long-range Linkage Disequilibrium in Cattle

The first data set used to measure LD in the dutch black-and-white population was a previously described granddaughter design (GDD), with 22 paternal half-sib families comprising a total of 949 bulls (Coppieters et al. 1998). This sample was genotyped for a battery of 284 autosomal microsatellites for a total of 276,048 genotypes. Figure 1 reports for each marker the heterozygosity measured in the 22 founder sires as well as the number of alleles observed in the overall population. The average heterozygosity as measured in the founder sires was $59 \%$, whereas the average number of alleles was 6.6. Linkage maps were constructed for all autosomes as described (Georges et al. 1995), yielding a total map length of $2702 \mathrm{cM}$ (Kosambi map) with an average between-marker interval of $13.4 \mathrm{cM}$. Order and distance between markers as well as estimates of total map length were in good agreement with Kappes et al. (1997).

The most likely linkage phase of the 22 foundersires and their respective sons was estimated for the 29 autosomes as described in Materials and Methods. The maternally inherited chromosomes of the sons were considered to be a representative sample of the dutch black-and-white breeding population. For dams having multiple sons in the GDD, only one of the sons was considered in the analysis. In total, we selected 581 


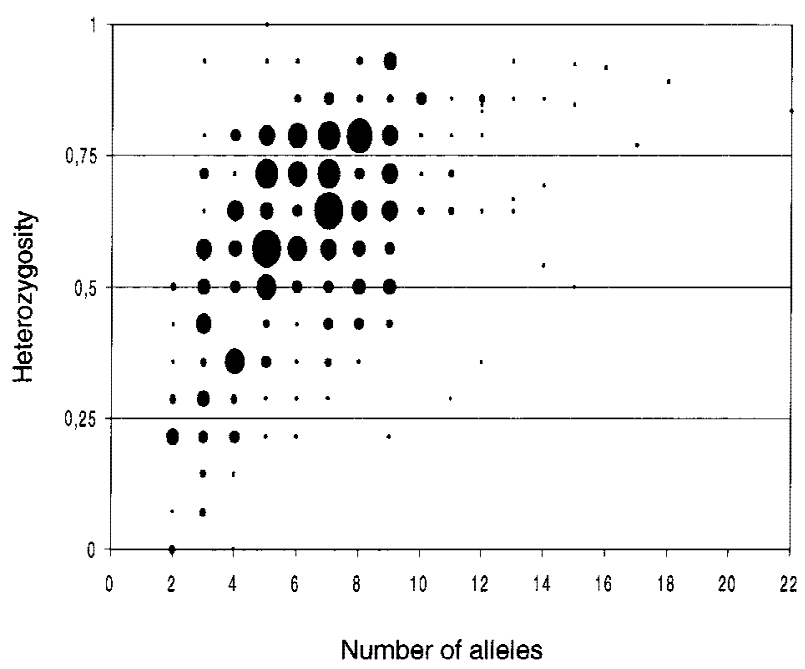

Figure 1 Relationship between the number of alleles and the heterozygosity observed in the dutch black-and-white population for the utilized panel of autosomal microsatellite markers $(n=284)$. The diameter of the bullet reflects the number (range: 1-9) of markers with corresponding heterozygosity and allele number.

such maternal "gametes" for further analysis. The corresponding genotypes were used to estimate LD between all 40,186 pairs of markers, using Lewontin's (1964) normalized $D^{\prime}$ measure (see Materials and Methods).

The extent of LD was first evaluated for syntenic marker pairs. Figure 2A shows the distribution of $D^{\prime}$ values as a function of genetic distance in centimorgans. $D^{\prime}$ averaged $50 \%$ for marker pairs $<5 \mathrm{cM}$ apart, decayed rapidly to values of the order of $16 \%$ for distances of $50 \mathrm{cM}$, and then reached a plateau slightly below $14 \%$ for more distant markers. The statistical significance of the corresponding LD, $\alpha$, was estimated by Monte-Carlo approximation of Fisher's exact test as described by Weir (1996). More specifically (see Materials and Methods), we examined the cumulative frequency distribution of $\alpha$ values for syntenic marker pairs grouped by distance in recombination units (Fig. 2C). All observed frequency distributions differed dramatically from that expected under the null hypothesis of linkage equilibrium $(P<0.001)$, clearly indicating that substantial levels of intrachromosomal LD can be captured with the utilized marker density, not only for closely linked, but even for the most distant, syntenic markers. Grouping marker pairs $<5 \mathrm{cM}$ apart in 1 -cM bins, indicates that average $D^{\prime}$ values continue to increase with decreasing distance between markers, providing no evidence for a saturation of the LD signal $<5$ cM (data not shown).

Intrigued by the long-range LD observed between syntenic markers, we then looked for possible gametic phase disequilibrium between nonsyntenic loci. Figure $2 \mathrm{~B}$ reports the frequency distribution of $D^{\prime}$ values mea-
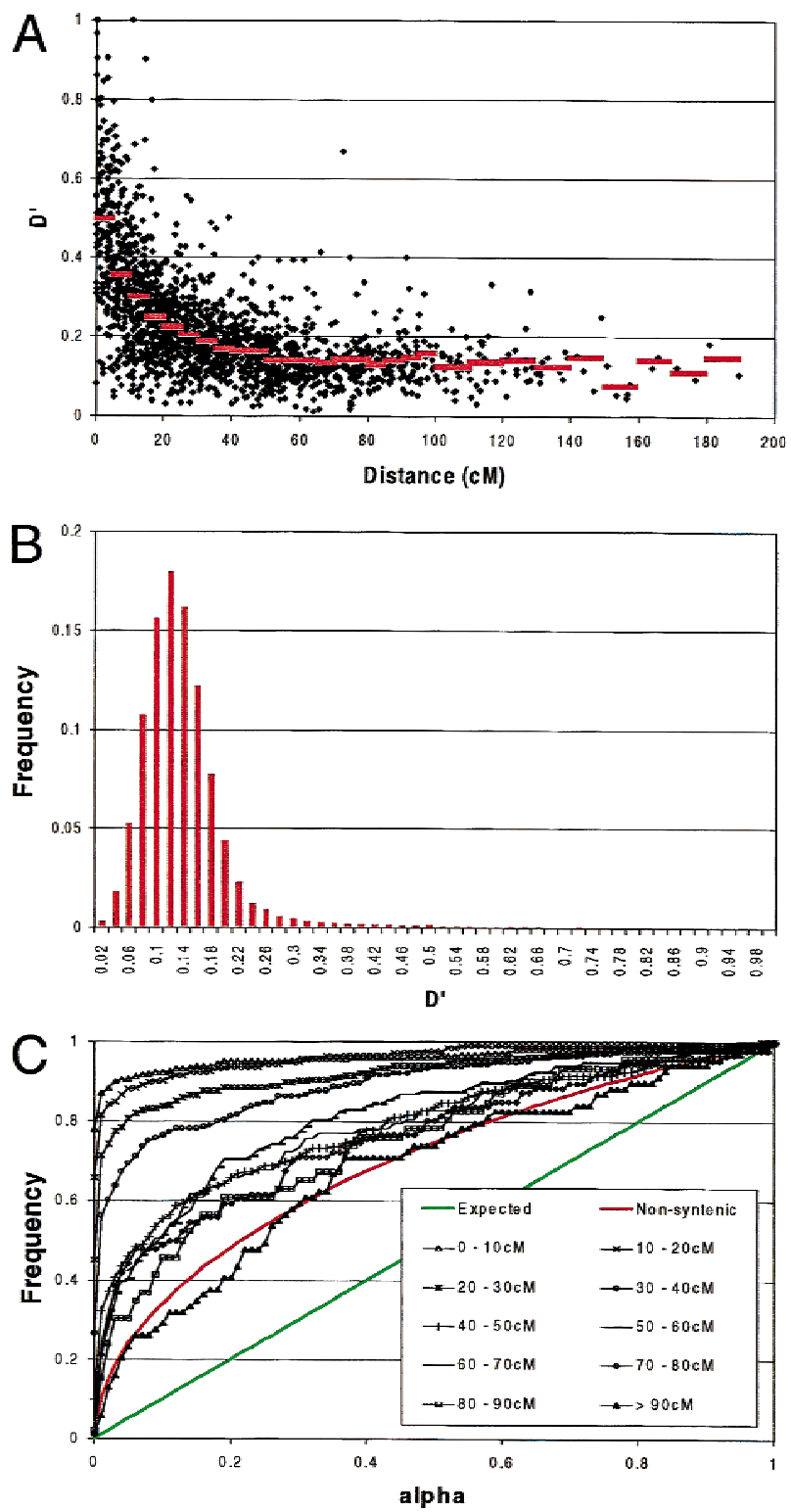

Figure 2 Real data-data set 1. (A) Distribution of $D^{\prime}$ values observed between syntenic marker pairs as a function of genetic distance in centimorgan (cM). The red lines correspond to average $D^{\prime}$ values for marker pairs sorted in $5 \mathrm{cM}$ bins $(0-50 \mathrm{cM})$ or 10 -cM bins (50-190 cM). (B) Frequency distribution of $D^{\prime}$ values observed for all nonsyntenic marker pairs. (C) Cumulative frequency distribition of $\alpha$ values. (Black) Pairs of syntenic markers grouped by genetic distance; (red) pairs of nonsyntenic markers; (green) expected distribution under random allelic assortment.

sured between nonsyntenic marker pairs. The average $D^{\prime}$ value was $12 \%$, therefore quite similar to the $D^{\prime}$ value found for distant $(>50 \mathrm{cM}$ ) though syntenic loci. Examination of the corresponding cumulative frequency distribution of $\alpha$-values (Fig. 2C) indicates that the observed gametic phase disequilibrium between nonsyntenic loci was highly significant as well $(P<0.001)$. 
The results described previously were obtained using gametes from so-called bull-dams, that is, elite cows selected to produce top bulls. One could argue that this so-called "active breeding population" is not representative of the breed in general. We therefore examined a second data set assumed to be more representative of the general population. We collected DNA from 627 cows, daughters of four sires, as well as from their respective dams in a large number of dutch herds. The four sires, all daughters, and their corresponding dams were genotyped for eight microsatellite markers located on different autosomes. 175 daughters and their dams were genotyped for an additional 19 markers, 16 of these located on chromosome 14 and three on chromosome six. We determined the genotype of the gamete transmitted to the daughter as well as its "complement" (see Materials and Methods), yielding a total of 1254 gametes. Figure 3 shows the distribution of $D^{\prime}$ values obtained for the 123 tests performed between syntenic markers (Fig. 3A) as well as for the 228 nonsyntenic tests (Fig. 3B). $D^{\prime}$ averaged $46 \%$ for marker pairs $<5 \mathrm{cM}$ apart, decaying to $24 \%$ on average for marker pairs at a distance of $30 \mathrm{cM}$ or more. The average $D^{\prime}$ value measured between nonsyntenic markers was $20 \%$, therefore even higher than the value observed with the bull-dam gametes. The departure from expectation proved to be highly significant $(P<0.001)$ for both syntenic and nonsyntenic marker pairs (Fig. 3C). Overall, these results provide strong evidence that long-range LD and gametic association between nonsyntenic loci is a genuine feature characterizing the dutch black-and-white dairy cattle population in general and not only the elite bull-dam population.

\section{Random Drift Accounts for Most \\ of the Observed Disequilibrium}

The observation of this unexpectedly high degree of linkage disequilibrium poses the question of its origin. It is well established from classical population genetics theory that drift (Hill and Roberston 1968; Ohta and Kimura 1969), migration (admixture) (e.g., Stephens et al. 1994), mutation, and selection (Bulmer 1971) generate linkage disequilibrium. Worldwide, the blackand-white dairy population counts $>25$ million animals. In the Netherlands only, the population of blackand-white cattle comprises 1.2 million lactating cows. Estimates of effective population size, however, yield numbers as low as 50 (Boichard 1996). This is primarily attributable to the widespread use of artificial insemination (A.I.) and the intense selection for increased milk production. As an example, in the Netherlands $95 \%$ of cows are bred by A.I. and the 10 top sires account for $40 \%$ of the inseminations.

To evaluate whether the population structure of the dutch black-and-white population alone could account for the observed linkage disequilibrium, we col-
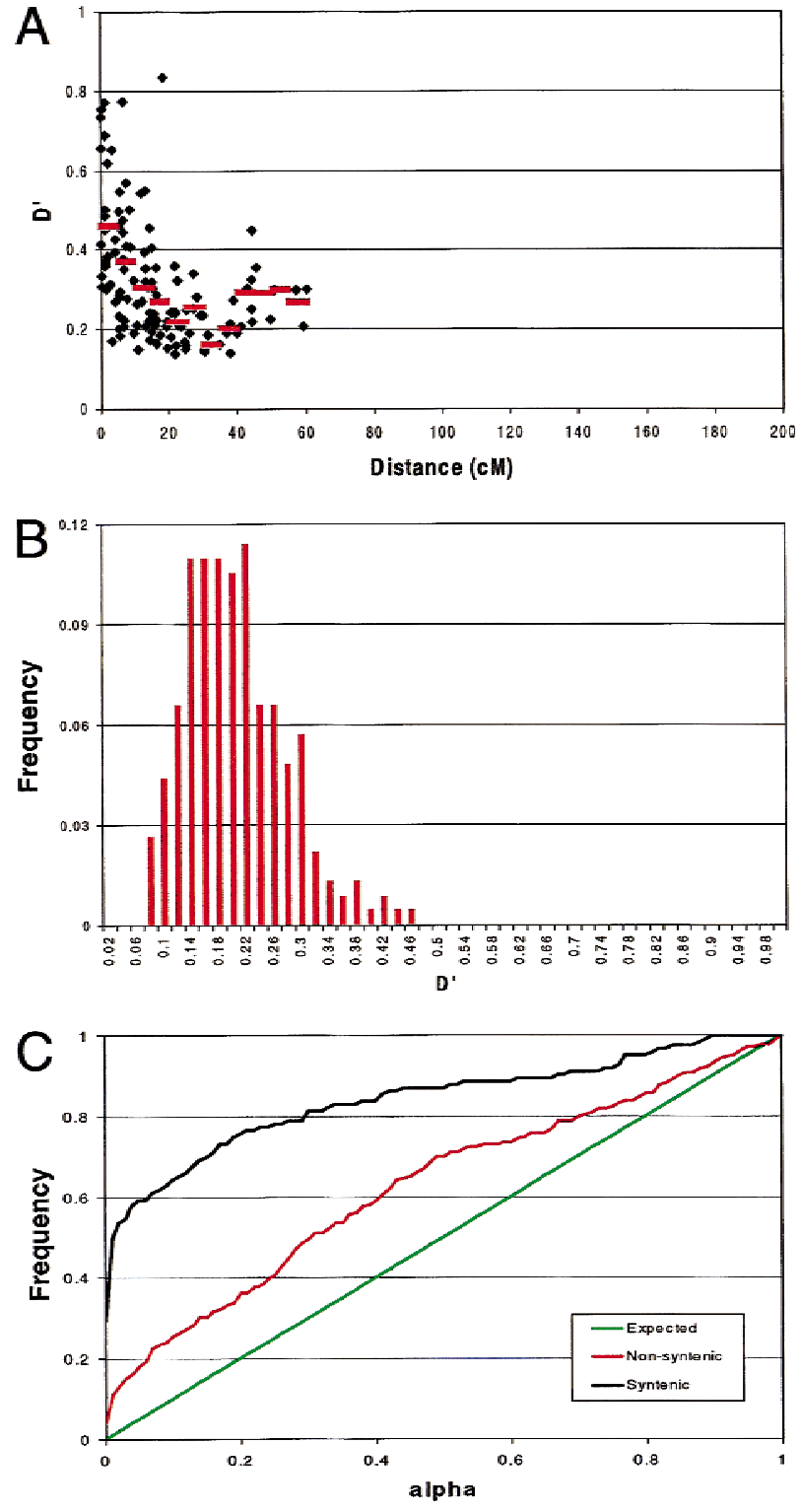

Figure 3 Real data-data set 2. (A) Distribution of $D^{\prime}$ values observed between syntenic marker pairs as a function of genetic distance in centimorgan (cM). The red lines correspond to average $D^{\prime}$ values for marker pairs sorted in 5-cM bins $(0-60 \mathrm{cM})$. (B) Frequency distribution of $D^{\prime}$ values observed for all nonsyntenic marker pairs. (C) Cumulative frequency distribition of $\alpha$-values. (Black) Pairs of syntenic markers; (red) pairs of nonsyntenic markers; (green) expected distribution under random allelic assortment.

lected the known genealogies of the studied bull-dams (data set 1 ). The average number of recorded ancestors per bull-dam was 40.4 , whereas up to 11 generations separated the bull-dams from their most distant ancestor. Based on the available pedigree data and assuming that the "founders" were unrelated, we estimated the average inbreeding coefficient, $F$, of the bull-dams at $1.3 \%$ (range: $0 \%-14 \%$ ), and their average kinship co- 
efficients, $f$, at $4 \%$ (range: $0 \%-57 \%$ ). We simulated the segregation in this pedigree material of 29 autosomes covered with markers mimicking the actual microsatellite map (see Material and Methods). A gamete was drawn at random from each bull-dam and the resulting collection of genotypes used to measure LD between syntenic markers as well as gametic phase disequilibrium between nonsyntenic markers (Fig. 4). $D^{\prime}$ values averaged 33\% for syntenic marker pairs <5 cM apart, that is, slightly lower than the values observed with the real data. As expected, $D^{\prime}$ values decreased with increasing distance between markers to plateau at a value of $14.7 \%$ for marker pairs $>50 \mathrm{cM}$ apart, therefore very similar if not slightly superior to the corresponding value of $13.8 \%$ obtained with the real genotypes (Fig. $4 \mathrm{~A}$ ). For nonsyntenic marker pairs, the average $D^{\prime}$ value was $12 \%$, therefore virtually identical to the value found with the real data set (Fig. 4B). The cumulative frequency distributions of $\alpha$ values were shown to depart very significantly from the distribution expected in case of linkage equilibrium $(P<0.001)$, both for syntenic marker pairs sorted by distance as well as nonsyntenic markers (Fig. 4C). These results therefore clearly indicated that the population structure alone suffices to generate substantial levels of both syntenic and nonsyntenic LD, very similar in magnitude to that observed with the real data. The most striking difference between the real and the simulated results are that for closely linked markers ( $<5 \mathrm{cM}$ apart) the average $D^{\prime}$ values are considerably higher for the real data (50\%) when compared with the simulated data (33\%). The intrinsic differences between the real and simulated data sets therefore seem to affect the level of disequilibrium between closely linked versus distant markers more profoundly. Note that despite the extensive pedigree recording that is customary in dairy cattle breeding, the available genealogies of the bull-dams are far from complete (see above). In the simulations, all "founder" chromosomes were assumed to be in linkage equilibrium (see Materials and Methods), which would be expected to reduce the overall level of LD when compared with the real data.

In addition to random drift, migration (admixture) is likely to have contributed to the observed levels of LD as well. The globalization of semen trade has caused considerable gene flow between demes, particularly from the United States to the rest of the world (e.g., Goddard 1992). Differences in allelic frequencies between demes could only have increased the level of LD.

\section{Lack of Evidence in Favor of the Bulmer Effect}

Selection is also predicted to cause gametic phase disequilibrium (Bulmer 1971). Contrary to the other factors that cause LD, however, selection will preferentially generate disequilibrium between loci influencing
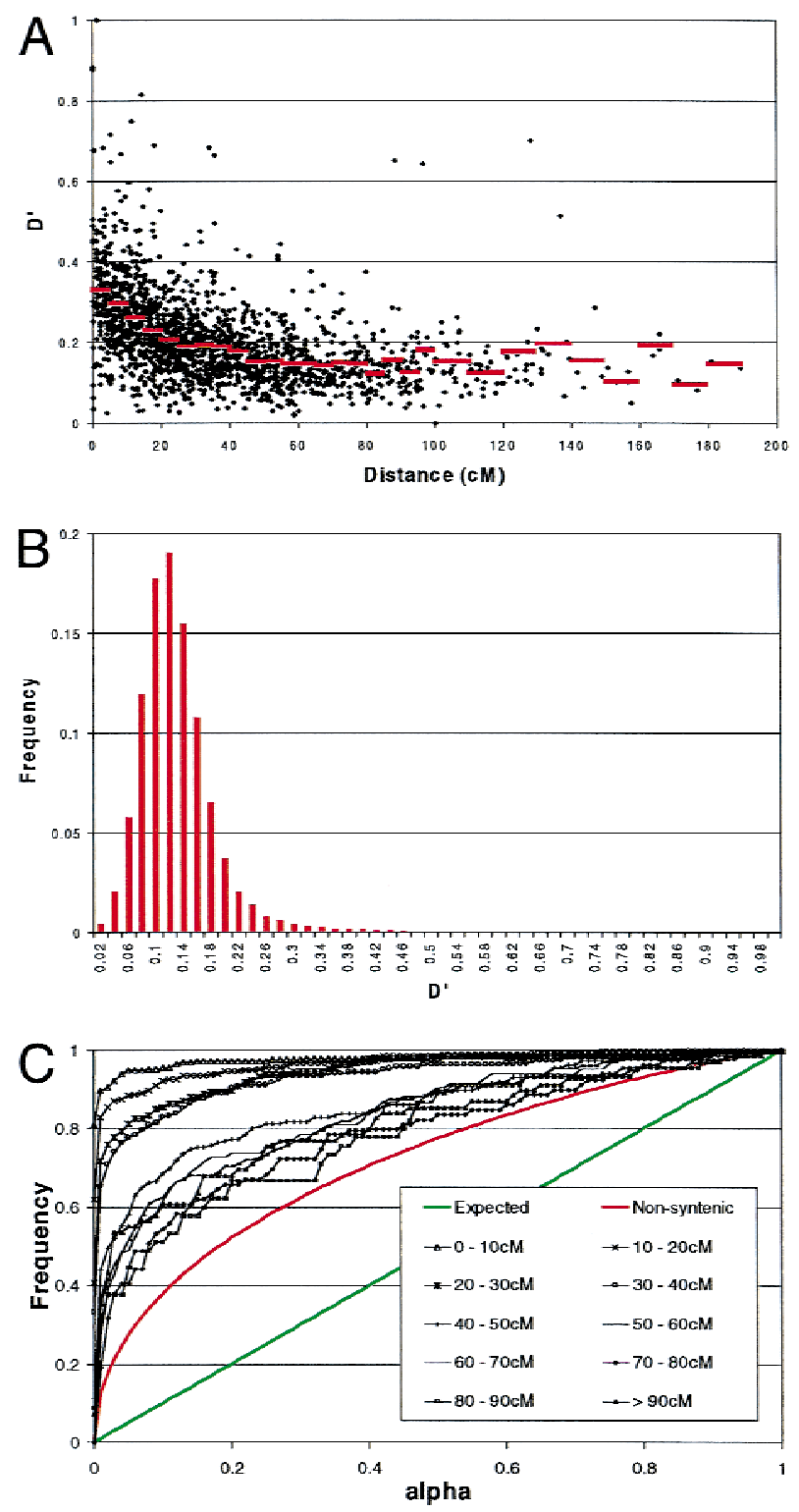

Figure 4 Simulated data. $(A)$ Distribution of $D^{\prime}$ values observed between syntenic marker pairs as a function of genetic distance in centimorgan (cM). The red lines correspond to average $D^{\prime}$ values for marker pairs sorted in $5-\mathrm{cM}$ bins $(0-50 \mathrm{cM})$ or $10 \mathrm{cM}$ bins (50-190 cM). (B) Frequency distribution of $D^{\prime}$ values observed for all nonsyntenic marker pairs. (C) Cumulative frequency distribition of $\alpha$ values. (Black) Pairs of syntenic markers grouped by genetic distance; (red) pairs of nonsyntenic markers; (green) expected distribution under random allelic assortment.

the selected phenotype. Directional and stabilizing selection for instance tend to generate negative gameticphase disequilibrium (alleles increasing the character value at one locus preferentially associated with alleles decreasing the character value at other loci and vice versa), whereas disruptive selection and directional selection on characters displaying certain patterns of epistasis will generate positive gametic-phase disequilibrium (Walsh and Lynch 1999). Therefore, if selec- 
tion were to contribute significantly to the observed genome-wide $\mathrm{LD}$, one would predict that $D^{\prime}$ values would not be distributed uniformly across the genomewide nonsyntenic LD map but would have a tendency to be higher for chromosome pairs harboring Quantitative Trait Loci (QTL) undergoing selection. Figure 5 illustrates the genome-wide LD map obtained with both the real (data set 1) and simulated data. We analyzed the nonsyntenic $D^{\prime}$ values using a linear model including the effect of the two corresponding chromosomes, as well as a term testing for the interaction between these chromosomes (see Materials and Methods). A significant interaction term would have been interpreted as being in favor of the Bulmer effect. As expected, the interaction term was not significant for the simulated data set $(P=0.97)$, as the simulation was performed in the absence of selection. This term was also not significant, however, with the real data $(P=0.98)$, therefore providing no evidence in favor of a strong contribution of selection to the gametic association observed between nonsyntenic marker loci. Examination of the genomic distribution of nonsyntenic marker pairs exhibiting $D^{\prime}$ values $>0.3$ did not point toward the preferential involvement of specific chromosomes, including those that are known to harbor QTL, influencing milk yield and composition (e.g., Georges et al. 1995; Coppieters et al. 1998).

Interestingly, the chromosome effects proved to be highly significant for both the real and simulated data sets $(P<0.0001)$. Despite the fact that $D^{\prime}$ is assumed to be a frequency-independent measure of LD (Hedrick 1987), we attribute this significant chromosome effect to differences in information content between markers.

\section{DISCUSSION}

Our results suggest that mapping strategies exploiting

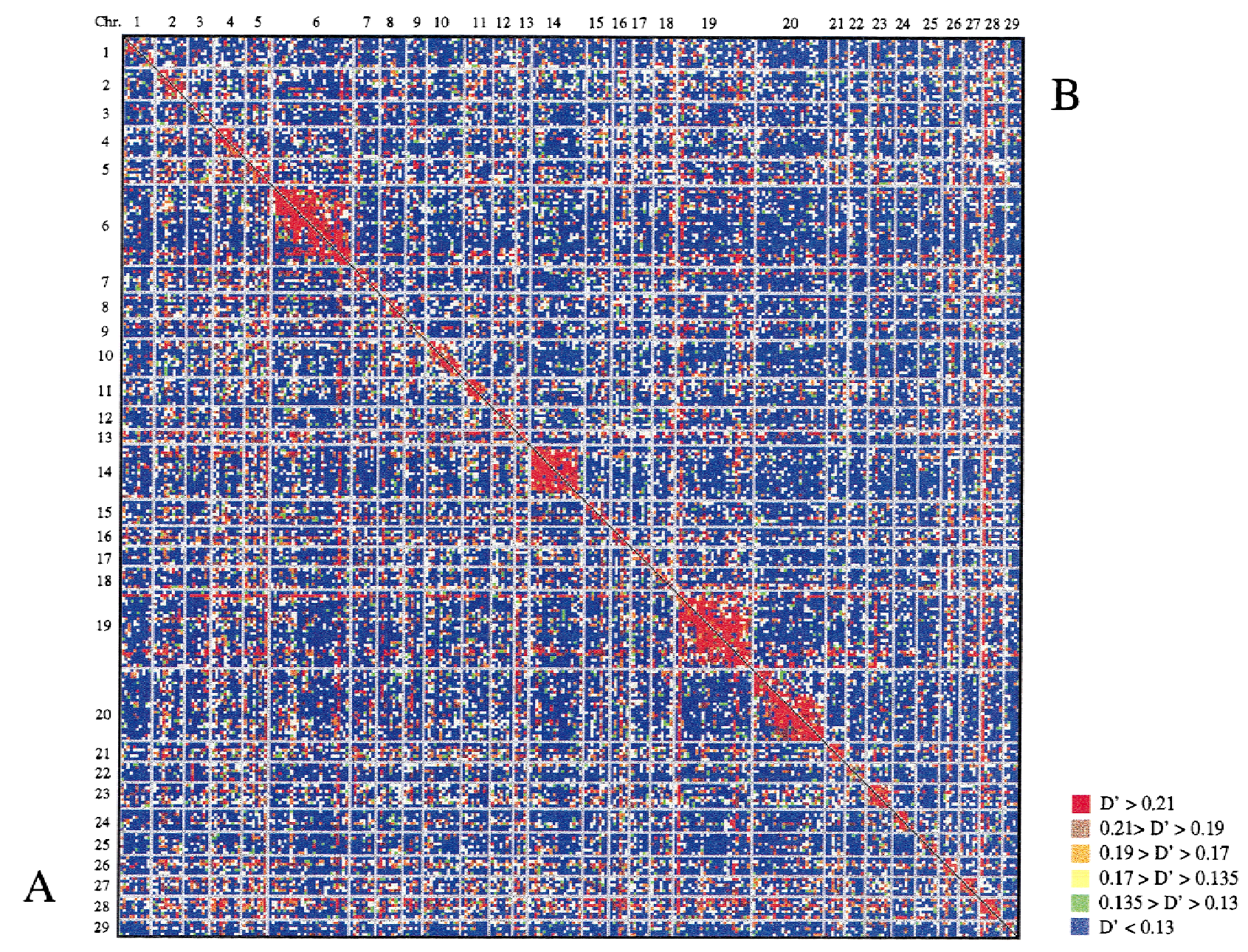

Figure 5 Genome-wide linkage disequilibrium map. Microsatellite markers are ordered along the $\mathrm{X}$ and $\mathrm{Y}$-axis by chromosome and order within chromosome. Every pixel reports the $D^{\prime}$ value for the corresponding marker pair using the shown color code. Pixels below the diagonal testing syntenic marker pairs correspond to the real data set $1(A)$, whereas pixels above this diagonal correspond to the simulated data set $(B)$.

\section{Genome Research}


LD may be particularly effective in dairy cattle (e.g., Charlier et al. 1996; Riquet et al. 1999). Whereas the present study concentrated on the dutch black-andwhite population, we predict that similar situations will be encountered in most other dairy cattle populations were A.I. is widespread. Contrary to the situation in the human where genome-wide LD mapping may require a marker density two orders of magnitude higher than that required for conventional linkage mapping (Kruglyak 1999), the available battery of $\cong 1,500$ microsatellites (Kappes et al. 1997) could be sufficient for first-pass LD screening in dairy cattle. The corollary of this observation, however, is that the mapping resolution to be gained from LD is likely to be limited in these populations as well. In this work, we still observed a considerable drop in $D^{\prime}$ values between 1 and $5 \mathrm{cM}$, suggesting that it should nevertheless be possible to achieve resolution down to the centimorgan level. Further analyses will be required to evaluate the benefit of LD mapping at the sub-centimorgan level in these populations.

Because of their higher mutation rate, the usefulness of microsatellite markers for LD mapping when compared with single nucleotide polymorphisms (SNPs) has been questioned by some. The extensive LD observed suggests, however, that in cattle populations Identity By Descent (IBD) chromosome segments will on average coalesce within considerably fewer generations when compared with most human populations. The relatively high mutation rate of microsatellite markers, susceptible to erase part of the LD signal in humans, is therefore less likely to cause a problem for LD mapping in cattle.

The common occurrence of gametic-phase disequilibrium between nonsyntenic loci raises serious concerns about the generation of false-positive results when using association studies as the only means to locate genes underlying complex traits in these populations. Preference should therefore be given to mapping methods that combine linkage and LD information. This could be achieved using a two-tiered approach in which the rough map position of the genes of interest is first determined by linkage analysis followed by their fine-mapping using LD. Alternatively, one could use approaches akin to the Transmission Disequilibrium Test (e.g., Spielman et al. 1993), which are simultaneously testing for linkage and LD.

The fact that we were not able to provide evidence in favor of the Bulmer effect, does not mean that it doesn't operate in the studied population. More refined methods are probably required to reveal the specific contribution of selection to the gametic-phase disequilibrium observed between chromosome regions harboring QTL. As several QTL influencing milk yield and composition have now been uncovered and marker haplotypes associated with specific QTL alleles are being detected (e.g., Riquet et al. 1999), such experiments should become feasible in the near future.

\section{MATERIALS AND METHODS}

\section{Genotype Determination}

Microsatellite genotypes, marker maps, and the most likely linkage phase of the founder sires were obtained as described (Georges et al. 1995). Assuming known linkage phase of the sires, we determined the most likely linkage phase of each offspring accounting for the marker genotype of the dam when available (data set 2) or marker allele frequencies in the general population when not (data set 1) (F. Farnir, unpubl.). For data set 1 , the maternal gametes transmitted to the sons were used to estimate LD. This yielded a sample of 581 gametes. For data set 2 , the maternal gametes transmitted to the daughters as well as the "complementary" gamete (the genotype of which could be inferred by substracting the genotype of the transmitted gamete from the known genotype of the dam) were used to estimate $\mathrm{LD}$. This yielded a sample of 1,254 gametes. Figure 6 summarizes the pedigree structure for data sets 1 and 2 .

\section{Measuring Linkage Disequilibrium}

Following Hedrick (1987), LD between two polyallelic loci $A$ and $B$ was measured as:

$$
D^{\prime}=\sum_{i=1}^{u} \sum_{j=1}^{v} p_{i} q_{j}\left|D^{\prime} i j\right|
$$

where $u$ and $v$ are the respective number of alleles at the two marker loci, $p_{i}$ and $q_{j}$ are the population frequencies of marker allele $i$ at locus $A$ and marker allele $j$ at locus $B$, and $\left|D^{\prime}{ }_{i j}\right|$ is the absolute value of Lewontin's (1964) normalized LD measure computed as:

$$
D_{i j}^{\prime}=\frac{D_{i j}}{D_{\max }}
$$

with:

$$
D_{i j}=x_{i j}-p_{i} q_{j}
$$

where $x_{i j}$ is the observed frequency of gametes $A_{i} B_{j}$, and $p_{i}$ and $q_{j}$ are the frequencies of alleles $A_{i}$ and $B_{j}$ respectively, and:

$$
D_{\max }=\left[\begin{array}{l}
\min \left[p_{i} q_{j,}\left(1-p_{i}\right)\left(1-q_{j}\right)\right] ; D_{i j}<0 \\
\min \left[p_{i}\left(1-q_{j}\right),\left(1-p_{i}\right) q_{j}\right] ; D_{i j}>0
\end{array}\right]
$$

The statistical significance $(\alpha)$ of the observed allelic association under the null hypothesis of random allelic assortment, was estimated by Monte-Carlo approximation of Fisher's exact test as described by Weir (1996). Briefly, assume a sample of $n$ gametes genotyped for marker loci $A$ and $B$ having respectively $u$ and $v$ alleles. The sample is fully characterized by allele counts $n_{i .}$. (locus $A$ ) and $n_{. j}$ (locus $B$ ) and haplotype counts $n_{i j}$, as illustrated in the following table for a simple example where locus $A$ and $B$ are characterized by three and two alleles, respectively. The probability of a given sample, $P$, can be computed as: 


\section{Data set 1}

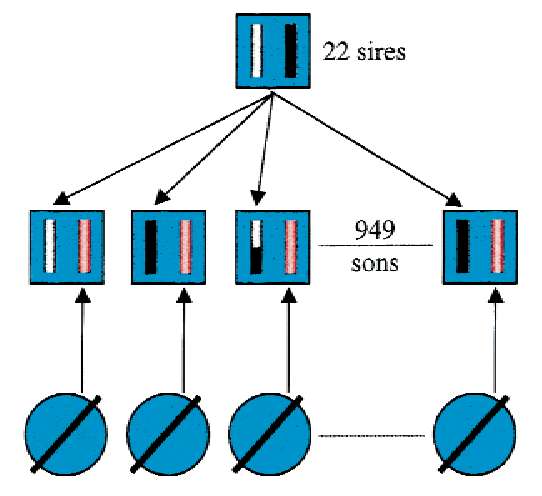

\section{Data set 2}

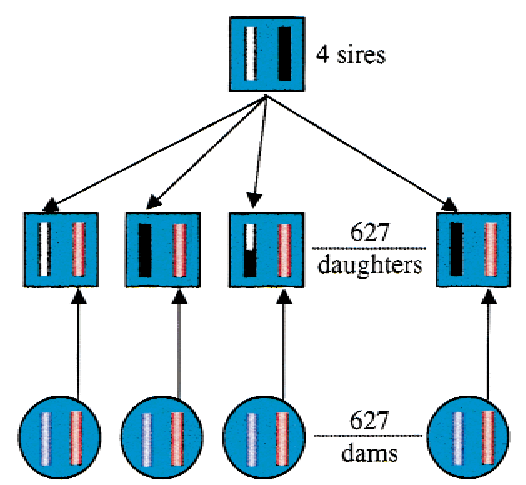

departure from expectation of the observed cumulative frequency distribution was estimated from the area bounded by the expected and observed lines. The corresponding surface was computed by numerical integration. The distribution of this area under the null hypothesis of random assortment was obtained by Monte-Carlo simulation $(1,000$ simulations) as well.

\section{Determination of Kinship and Inbreeding Coefficients \\ Pedigree information was directly obtained from the NRS (Arnhem, The Netherlands). Kinship and in- breeding coefficients were com- puted using PROC INBREED from the SAS package version 6.12 . \\ Simulating Chromosome Segregation in the Bull-Dam Genealogy}

Figure 6 (Left) Data set 1: Data set 1 corresponds to a GDD described previously, that is, a series (22) of paternal half-brother families with their sires for a total of $22+949$ bulls (Coppieters et al. 1998). The dams of data set 1 are not genotyped. The marker linkage phase of the founder sires are determined from the genotypes of their respective sons as described (Georges et al. 1995). Assuming known marker phase of the sire, the most likely genotypes of paternal (black, white, or recombinant) and maternal (red) gametes transmitted to the son can be inferred. The genotypes of the maternal gametes (red) were used to measure LD. (Right) Data set 2: Data set 2 corresponds to a daughter design, that is, a series (4) of paternal half-sister families with their sires for a total of 624 daughters. The 624 dams of data set 2 were genotyped as well. The marker-linkage phase of the founder sires are determined from the genotypes of their respective daughters as described, exploiting the available genotype information from the dams (Georges et al. 1995). Assuming known marker phase of the sire, the most likely genotypes of paternal (black, white, or recombinant) and maternal (red) gametes transmitted to the daughters can be inferred. The genotypes of the maternal gametes (red) as well as their complement (blue) were used to measure LD.

\begin{tabular}{|l|c|c|c|c|l|}
\hline \multicolumn{2}{|c|}{} & \multicolumn{3}{|c|}{ Locus A } & \multirow{3}{*}{} \\
\cline { 3 - 6 } \multicolumn{2}{|c|}{} & 1 & 2 & 3 & \\
\hline \multirow{2}{*}{$\begin{array}{l}\text { Locus } \\
\text { B }\end{array}$} & 1 & $n_{11}$ & $n_{21}$ & $n_{31}$ & $n_{.1}$ \\
\cline { 2 - 6 } & 2 & $n_{12}$ & $n_{22}$ & $n_{32}$ & $n_{.2}$ \\
\hline & & $n_{1 .}$ & $n_{2 .}$ & $n_{3 .}$ & $n$ \\
\hline
\end{tabular}

$$
P=\frac{\prod_{i=1}^{u} n_{i} ! \prod_{j=1}^{v} n_{j} !}{n ! \prod_{i=1}^{u} \prod_{j=1}^{v} n_{i j} !}
$$

The value of $\alpha$ for a given marker pair corresponds to the proportion of all possible tables with same allele counts $\left(n_{i \text {. }}\right.$ and $n_{. j}$ ) that have equal or lower $P$. $\alpha$ can be estimated by simulating such tables under the hypothesis of random assortment and counting the proportion of tables that have equal or lower $P$ than the real sample. In this study, the estimates of $\alpha$ were based on the simulations of 16,590 such tables.

The $\alpha$ values generated as described do not account for the large number $(40,186)$ of tests performed. Rather than applying a Bonferroni correction on individual $\alpha$ values (and essentially lose all power to detect non random assortment), we compared the observed cumulative frequency distribution of $\alpha$ values with that expected under the null hypothesis of random allelic assortment. The statistical significance of the and assuming linkage equilibrium between markers. Founder chromosomes were then allowed to segregate within the pedigree, including recombination with their homologs at a rate determined by the genetic distance between adjacent markers.

\section{Testing for the Bulmer Effect}

$D^{\prime}$ values for nonsyntenic marker pairs were analyzed with the following linear models:

$$
\begin{aligned}
& \text { Model I: } D_{n(i), m(j)}^{\prime}=\mu+C_{i}+C_{j}+\varepsilon_{n, m} \\
& \text { Model II: } D_{n(i), m(j)}^{\prime}=\mu+C_{i}+C_{j}+C_{i} * C_{j}+\varepsilon_{n, m}
\end{aligned}
$$

where:

$D_{n(i), m(j)}^{\prime}$ is the $D^{\prime}$ value computed for marker $n$, located on chromosome $i$, and marker $m$, located on chromosome $j ; \mu$ is the average $D^{\prime}$ value over all marker pairs; $C_{i}$ and $C_{j}$ are the effects of chromosomes $i$ and $j$ respectively; $C_{i}{ }^{*} C_{j}$ is the interaction effect between chromosomes $i$ and $j$, and $\epsilon_{\mathrm{n}, \mathrm{m}}$ is the error term.

Chromosome and interaction effects were estimated using standard least square methodology (Searle 1971).

The significance of the chromosome effects was estimated from: 


$$
\frac{\operatorname{SSR}_{\text {Model I }} *\left(N-r\left(X_{I}\right)\right)}{\operatorname{SSE}_{\text {Model I }} *\left(r\left(X_{I}\right)-1\right)}=F_{r\left(X_{I}\right)-1, N-r\left(X_{I}\right)}
$$

where $S S R$ is the sum of squares caused by the chromosome effects, SSE is the residual sum of squares, $N$ is the number of nonsyntenic marker pairs, and $r\left(X_{I}\right)$ is the rank of the incidence matrix for Model I, that is, 29.

The significance of the interaction term was estimated from:

$$
\frac{\left(S S E_{\text {ModelI }}-S S E_{\text {ModelII }}\right) *\left(N-r\left(X_{I I}\right)\right)}{S S E_{\text {ModelII }} *\left(r\left(X_{I I}\right)-r\left(X_{I}\right)\right)}=F_{r\left(X_{I I}\right)-r\left(X_{I}\right), N-r\left(X_{I I}\right)}
$$

where $r\left(X_{I I}\right)$ is the rank of the incidence matrix for Model II, that is, 406 .

\section{ACKNOWLEDGMENTS}

This work was funded by grants from CR Delta (Arnhem, The Netherlands), Livestock Improvement Corporation (Hamilton, New Zealand), the Vlaamse Rundvee Vereniging, the Ministère des Classes Moyennes et de l'Agriculture, Belgium and E.U. Grants B104-CT95-0073 and PL970471. We are grateful to Chris Schrooten for providing us with the pedigree information, as well as Jos Koopman and Didier Boichard for fruitful discussions.

The publication costs of this article were defrayed in part by payment of page charges. This article must therefore be hereby marked "advertisement" in accordance with 18 USC section 1734 solely to indicate this fact.

\section{REFERENCES}

Boichard, D. 1996. Analyse généalogique des races bovines laitières francaises. INRA Prod. Anim. 9: 323-335.

Bulmer, M.G. 1971. The effect of selection on genetic variability. Am. Nat. 105: 201-211.

Charlier, C., F. Farnir, P. Berzi, P. Vanmanshoven, B. Brouwers, and M. Georges. 1996. IBD mapping of recessive traits in livestock: Application to map the bovine syndactyly locus to chromosome 15. Genome Res. 6: 580-589.

Coppieters, W., J. Riquet, J.-J. Arranz, P. Berzi, N. Cambisano, B. Grisart, L. Karim, F. Marcq, P. Simon, P. Vanmanshoven, D. Wagenaar, and M. Georges. 1998. A QTL with major effect on milk yield and composition maps to bovine chromosome 14 . Mamm. Genome 9: 540-544.

Crouau-Roy, B., S. Service, M. Slatkin, and N. Freimer. 1996. A fine-scale comparison of the human and chimpanzee genomes: Linkage, linkage disequilibrium and sequence analysis. Hum. Mol. Genet. 5: 1131-1137.

Georges, M., D. Nielsen, M. Mackinnon, A. Mishra, R. Okimoto, A.T. Pasquino, L.S. Sargeant, A. Sorensen, M.R. Steele, X. Zhao, J.E. Womack, and I. Hoeschele. 1995. Mapping quantitative trait loci controlling milk production by exploiting progeny testing. Genetics 139: 907-920.
Goddard, M.E. 1992. Optimal effective population size for the global population of black and white dairy cattle. J. Dairy Sci. 75: 2902-2911.

Hästbacka, J., A. de la Chapelle, I. Kaitila, P. Sistonen, A. Weaver, and E. Lander. 1992. Linkage disequilibrium mapping in isolated founder populations: diastrophic dysplasia in Finland. Nature Genet. 2: 204-211.

Hedrick, P.W. 1987. Gametic diequilibrium measures: Proceed with caution. Genetics 117: 331-341.

Hill, W.G. and A. Robertson. 1968. Linkage disequilibrium in finite populations. Theor. Appl. Genet. 38: 226-231.

Kappes, S.M., J.W. Keele, R.T. Stone, R.A. McGraw, T.S. Sonstegard, T.P.L. Smith, N.L. Lopez-Corrales, and C.W. Beattie. 1997. A second-generation linkage map of the bovine genome. Genome Res. 7: 235-249.

Kruglyak, L. 1999. Prospects for whole-genome linkage disequilibrium mapping of common disease genes. Nature Genet. 22: $139-144$.

Laan, M. and S. Pääbo. 1997. Demographic history and linkage disequilibrium in human populations. Nature Genet. 17: 435-438.

Lewontin, R.C. 1964. The interaction of selection and linkage. I. General considerations; heterotic models. Genetics 49: 49-67.

Nickerson, D.A., S.L. Taylor, K.M. Weiss, A.G. Clark, R.G. Hutchinson, J. Stengard, V. Salomaa, E. Vartiainen, E. Boerwinkle, and C.F. Sing. 1998. DNA sequence diversity in 19.7 $\mathrm{kb}$ region of the human lipoprotein lipase gene. Nature Genet. 19: $233-240$.

Ohta, T. and M. Kimura. 1969. Linkage disequilibrium at steady state determined by random genetic drift and recurrent mutation. Genetics 63: 229-238.

Riquet, J., W. Coppieters, N. Cambisano, J.-J. Arranz, P. Berzi, S. Davis, B. Grisart, F. Farnir, L. Karim, M. Mni, P. Simon, J. Taylor, P. Vanmanshoven, D. Wagenaar, J.E. Womack, and M. Georges. 1999. Identity-by-descent fine-mapping of QTL in outbred populations: Application to milk production in dairy cattle. Proc. Natl. Acad. Sci. 96: 9252-9257.

Searle, S.R. 1971. Linear models. John Wiley \& Sons Inc., New York, London, Sydney, Toronto.

Spielman, R.S., R.E. Ginnis, and W.J. Ewens. 1993. Transmission test for linkage disequilibrium: The insulin gene region and insulin-dependent diabetes mellitus (IDDM). Am. J. Hum. Genet. 52: $506-516$.

Stephens, J.C., D. Briscoe, and S.J. O’Brien. 1994. Mapping by admixture linkage disequilibrium in human populations: Limits and guidelines. Am. J. Hum. Genet. 55: 809-824.

Talbot, C.J., A. Nicod, S.S. Cherny, D.W. Fulker, A.C. Collins, and J. Flint. 1999. High-resolution mapping of quantitative trait loci in outbred mice. Nature Genet. 20: 305-308.

Terwilliger, J.D. and K.M. Weiss. 1998. Linkage disequilibrium mapping of complex disease: Fantasy or reality? Curr. Opin. Biotechnol. 9: 578-594.

Walsh, B.B. and B. Lynch. 1999. Evolution and selection of quantitative traits. http://nitro.biosci.arizona.edu/zbook/ volume_2/vol2.html.

Weir, B.S. 1996. Genetic data analysis II. Methods for discrete population genetic data. Pp. 117. Sinauer Associates, Inc. Publishers, Sunderland, MA.

Received September 10, 1999; accepted in revised form December 9, 1999. 


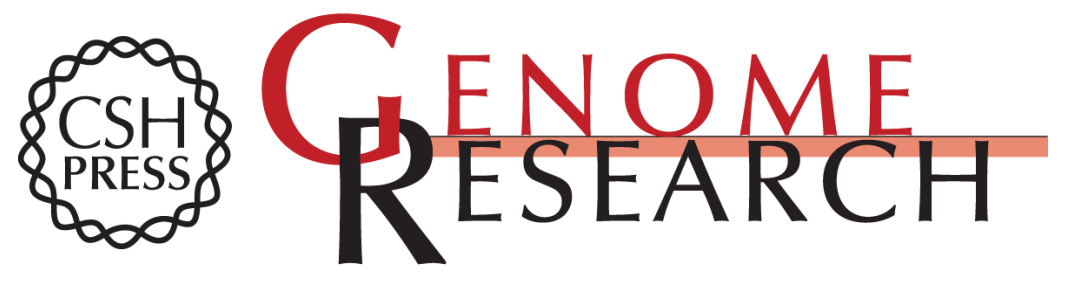

\section{Extensive Genome-wide Linkage Disequilibrium in Cattle}

Frédéric Farnir, Wouter Coppieters, Juan-José Arranz, et al.

Genome Res. 2000 10: 220-227

Access the most recent version at doi:10.1101/gr.10.2.220

References This article cites 21 articles, 7 of which can be accessed free at:

http://genome.cshlp.org/content/10/2/220.full.html\#ref-list-1

License

Email Alerting Receive free email alerts when new articles cite this article - sign up in the box at the Service top right corner of the article or click here.

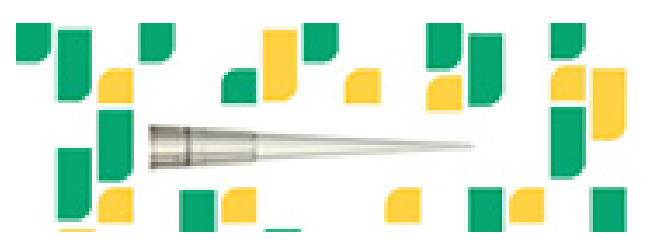

To subscribe to Genome Research go to: https://genome.cshlp.org/subscriptions 